\title{
A 30 años del golpe militar en Argentina: aproximaciones a la historia del Trabajo Social
}

\section{Shirly J. Mollo}

\section{0 anos do golpe militar na Argentina:
aproximações com a história do Serviço Social \\ 30 anos do golpe militar na Argentina:
aproximações com a história do Serviço Social}

30 Years after the military coup in Argentina:
approximations with the history of Social Work

30 Years after the military coup in Argentina:
approximations with the history of Social Work

Licenciada en Trabajo Social.

Profesora en la Universidad Nacional de Rosario, Argentina.

\section{Carina B. Molio}

Licenciada en Trabajo Social

Mestre y Doctora en Servicio Social.

Pós-doutorado em Serviço Social, PUC/ SP.

Profesora en la Universidade Federal de Juiz de Fora, Brasil.

\section{esumen}

Este artículo procura realizar una breve reconstrucción histórica del Trabajo Social, (sea del ámbito académico, sea de las organizaciones profesionales de los trabajadores sociales) durante el período comprendido entre los años 1960 hasta los días actuales. Como forma de abordar el objeto de estudio, se recurre a investigaciones que traten de la temática, a documentos escritos y a archivos. Las 26 entrevistas realizadas a trabajadores sociales que tuvieron un papel protagónico durante este período, se constituyeron en la llave heurística fundamental. En la primer parte del artículo es trabajado el período que antecede a la dictadura de 1976. En la segunda parte se aborda el período comprendido entre 1976-1983. El tercer punto trabaja sobre la democratización para finalmente puntualizar algunos desafíos y propuestas para pensar el Trabajo Social latinoamericano.

Palabras clave: Trabajo Social, historia, organización profesional.

\section{esumo}

Este artigo procura realizar uma breve reconstrução histórica do Serviço Social, (seja do âmbito acadêmico, seja das organizações profissionais dos assistentes sociais) durante o período compreendido entre os anos 1960 e os dias atuais. Como forma de abordar o objeto de estudo, recorreu-se a pesquisas que tratassem da temática, a documentos escritos e a arquivos. As 26 entrevistas realizadas com trabalhadores sociais, que foram protagonistas durante este período, constituíram-se na chave heurística fundamental. Na primeira parte do artigo trabalhase sobre o período que antecede à ditadura militar iniciada em 1976. Na segunda parte dedica-se ao período compreendido entre 1976-83. Na terceira, focaliza-se o período da democratização para, finalmente, particularizar alguns desafios e propostas para pensar o Serviço Social latinoamericano.

Palavras-chave: Serviço Social, história, organização profisssional.

\section{bstract}

This article conducts a brief historical reconstruction of Social Work (in both the academic realm as well as that of the professional organizations of social workers) from the 1960's until today. Research focused on documents and historic files. The 26 interviews conducted with social workers that had a protagonistic role in this period, constituted the principal heuristic base. The first part of the article deals with the period before the dictatorship of 1976. The second part concerns the period from 1976-1983. The third portion looks at democratization. It concludes by identifying some challenges and proposals that face Latin American social work.

Key words: Social Work, history, professional organization. 


\section{Introducción}

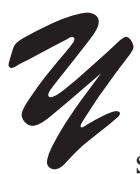
a han pasado 30 años del último golpe militar que azotó a la sociedad argentina; 30 años de una historia que viene siendo revisasada intensamente, 30 años en los que se siguen buscando respuestas, 30 años que a veces parecen que no hubiesen pasado. Sin embargo, el tiempo ha pasado y consideramos que la historia nos tiene que servir de lección y solamente nos puede enseñar, en la medida que aprehendamos críticamente lo que ha acontecido. Solamente seremos capaces de entender el presente, si comprendemos como llegamos a él, es decir, si comprendemos que el presente es la síntesis, la saturación de los acontecimientos pasados. Considerando estos asuntos, y preocupados con el presente, pero sobre todo con el futuro, es que nos proponemos repensar algunas cuestiones que hicieron y hacen parte del Trabajo Social argentino.

\section{Vísperas de la tragedia}

Cuando nos preguntamos qué acontecía con el Trabajo Social en el período anterior al golpe de Estado de 1976, especialmente en el ámbito académico y en las organizaciones profesionales, procuramos encontrar estas respuestas dentro de una historia social más amplia; dentro de los procesos sociales donde se inserta la profesión. Procurando conocer cuales fueron sus particularidades, y considerando el escaso material escrito sobre esta temática específica, ${ }^{1}$ entrevistamos a trabajadores sociales que tuvieron un papel protagónico durante ese período, es decir, que de alguna forma fueran representantes de su generación. Esta búsqueda no fue casual ni caprichosa, ella se debió a que gran parte de los archivos del período fueron quemados, pero sobre todo, porque entendemos que de las "experiencias vividas" podemos recuperar lo individual y lo colectivo de una generación que fue masacrada.

Como diría Benjamín (1994) recurrimos a las narrativas, a las experiencias que son transmitidas por el relato oral buscando en ellas lo que existe de colectivo en la memoria individual. Siguiendo esta línea de análisis, recuperamos parte de las historias de trabajadores sociales argentinos que participaron activamente de la vida política del país, o que tuvieron un rol protagónico en las organizaciones profesionales (especialmente en la Asociación de Asistentes Sociales o en el Colegio de Trabajadores Sociales). Nos circunscribiremos a lo acontecido en la ciudad de Rosario, provincia de Santa Fe, ya que gran parte de las entrevistas fueron realizadas en esta ciudad, aunque en diferentes momentos y en diferentes investigaciones ${ }^{2}$.

Entrevistamos principalmente a representantes de una generación que vivió directamente el golpe militar de 1976, que tenía entre 20 y 30 años en ese período, y que "experimentó" lo que fue llamado como "revolución cultural", una generación que fue influenciada por algunos acontecimientos internacionales ${ }^{3}$ que parecían indicar la posibilidad cercana de la transformación social.

Algunos de nuestros entrevistados comenzaron a estudiar a finales de la década del 1950, momento en que comenzaban a sentirse los cambios que habían sido introducidos a partir de la propuesta "modernizadora", que sintonizaba con el modelo económico desarrollista. En este sentido concordamos con Sigal (2002, p. 21) cuando señala que los tiempos de la universidad pueden ser comprendidos a través de los tiempos políticos, ya que existe una sintonía entre ambos: "La historia política de la Argentina proporciona una periodización posible de la historia de sus Universidades. No es naturalmente la única, pero es incontestable."

La teoría de la modernización venía generando una amplia aceptación dentro de la universidad, siendo uno de sus exponentes Gino Germani. Como contrapartida de esta teoría de explicar la sociedad, comenzaban a generarse otras formas basadas en diferentes teorías, sobre todo la teoría de la Dependencia. El marxismo, con las más "variadas interpretaciones" medio académico, denominado por varios autores como marxismo academicista ${ }^{5}$.

De modo muy sumario podríamos afirmar que el Trabajo Social veía, en el desarrollo de la comunidad, el "método apropiado" para desenvolver las capacidades de los hombres $^{6}$, lo que permitía superar la pobreza y por lo tanto lograr el buscado desarrollo social; se entendía que se trataba sobre todo de un cambio cultural, que posibilitaría traspasar el atraso de los países "subdesarrollados". Esta nueva "forma de comprender" el Trabajo Social, no llegó a romper con la visión tradicional ${ }^{7}$ del Trabajo Social, ni a proponer una ruptura con la visión del caso social individual. El "proyecto" modernizador no fue específico de la Argentina; en realidad abarcó gran parte de Latinoamérica, así como otros países del mundo capitalista ${ }^{8}$. En el caso de la Argentina, según consta en el informe que realizó la trabajadora social chilena Maidagan de Ugarte, durante su "misión" representando a la ONU en 1957, los planes de estudios de las Escuelas de Trabajo Social no se adaptaban a los "ideales de la modernización", por lo que se recomendó la creación de una nueva Escuela de Trabajo Social en la ciudad de Buenos Aires (ALAYÓN, 1978). El Instituto de Servicio Social ${ }^{9}$ creado en 1959 y cerrado en 1969 fue quien mejor expresó la visión modernizadora durante este período, y fue de allí que nació el grupo $\mathrm{ECRO}^{10}$.

En el momento en que se cerraba este Instituto, ocurría uno de los mayores movimientos de protesta vividos en la Argentina hasta esta fecha: los levantamientos populares conocidos como el "Rosariazo" y el "Cordobazo" en los cuales se unieron el movimiento estudiantil y el movimiento obrero ${ }^{11}$. En ese momento histórico presidía la república el dictador 
Onganía ${ }^{12}$. Sin duda el clima de politización vivenciada se contraponía con el modelo que se buscaba implantar.

La universidad había sido intervenida en 1966 con un grado de violencia que era impensable tiempos atrás ${ }^{13}$, deteniendo alumnos y profesores.La renuncia de los profesores fue masiva. Equipos enteros de profesores e investigadores dejaron la universidad e inclusive el país y se dirigieron, especialmente, hacia las universidades chilenas y venezolanas, en menor medida a Brasil y a Uruguay, como también hacia los Estados Unidos y Europa. Así recomenzaba un nuevo proceso de aniquilamiento de la educación pública en la Argentina.

Lo paradójico de esta época es que, cuanto más se "clausuraba" el escenario político, más movilización generaba; es decir, a pesar del fin de la autonomía universitaria, se ganaba una mayor autonomía de los actores universitarios. Muestra de ello son los nuevos centros de investigación que fueron creados a partir de 1966, que se constituyeron en medios de supervivencia intelectual y material (SIGAL, 2002). También comenzaron a proliferar los grupos de estudio, que fueron bautizados como "la universidad de las catacumbas".

Los pequeños grupos de estudio, fuera de los ámbitos de la universidad, fueron ganando espacio; participaban principalmente estudiantes y las discusiones giraban en torno a cuestiones académicas, sin embargo, con el cierre del debate político y del espacio público, en muchos casos estos grupos, terminaron siendo una vía de ingreso a la militancia política ${ }^{14}$, cambiando las preocupaciones estrictamente académicas por aquellas más relacionadas con la realidad social y política.

Fue en este período, donde las preocupaciones teóricas y las preocupaciones políticas comenzaron a confluir, llegando a su "auge" en 1973, momento en que se retoman las elecciones democráticas. No casualmente, durante estos años existió una suerte de "fusión" entre Trabajo Social y militancia política, sin conseguir distinguir donde comenzaba uno y donde la otra.

Sintonizados con los cambios que se iban produciendo, algunas Escuelas de Trabajo Social comenzaron a cambiar sus planes de estudios en 1969, pero fue sobre todo a partir de 1973 que estos comenzaron a modificarse. Entretanto es difícil evaluar el alcance de estos cambios, ya que no conocemos ningún estudio específico. Otro obstáculo es la diversidad de las Escuelas de Trabajo Social en la Argentina en este período, ya que en algunos casos dependían de la universidad, y en otros estaban dentro de los ámbitos ministeriales, sobre todo dentro de los ámbitos de los Ministerios de Acción Social, de Bienestar Social o de Salud Pública (provinciales o nacionales), como también dentro de instituciones privadas. La duración de las carreras tampoco fue homogénea, siendo que poseían períodos de 4 o de 3 años; algunas instituciones daban el título de Trabajador Social y otras de Asistente Social, en fin, no existía un plan de estudios único donde pudiesen convergir los ejes de formación de las diversas escuelas.

Lo que queda claro es que en este corto período de tiempo, comienza a producirse una suerte de inflexión dentro del Trabajo Social argentino ${ }^{15}$. Esto se hace evidente tanto por las publicaciones de la época, sea en los libros especializados, como en las dos revistas nacionales (Hoy en el Trabajo Social y Selecciones del Trabajo Social) que tenían circulación internacional, como por los congresos y encuentros latinoamericanos, panamericanos y regionales que eran muy frecuentes en ese momento. Una de las preocupaciones que guiaban a estos actores, muchos de los cuales se nucleaban dentro del movimiento de la reconceptualización, fue la creación de un Trabajo Social verdaderamente latinoamericano. De esta forma, se fueron fundando las bases, de lo que Netto (1996) denominó como el proceso de intención de ruptura. Los organismos internacionales, como el Instituto de Solidaridad Internacional (ISI), pero sobre todo los latinoamericanos, también comenzaron a ganar una fuerte influencia por entonces, primero a través de la Asociación Latinoamericana de Escuelas de Servicio Social (ALAESS, creado en 1965) $)^{16}$, y luego por el Centro Latinoamericano de Trabajo Social (CELATS, 1975).

Las universidades argentinas se multiplicaron durante este período, como muestra el artículo denominado "Situación de las Escuelas de Servicio Social argentinas" publicado en la Revista Selecciones del Servicio Social, número $20^{17}$. Se esperaba que aquellas Escuelas de Servicio Social que dependían de los ámbitos ministeriales, pasasen a depender de las universidades y así, integrasen la Política Nacional de Educación Superior.

Desde finales de 1974, el espacio público se fue cerrando cada vez más, y la sociedad vivió en un clima de militarización. Los grupos paramilitares, especialmente la triple A (alianza anticomunista argentina), intervenía dentro de las universidades así como en la vida pública, claro que de forma enmascarada; lo cierto es que a finales de 1975, el miedo había tomado gran parte de la sociedad. Muchos de los profesores y alumnos de la escuela de Trabajo Social de Rosario, comenzaron a sufrir amenazas anónimas y el clima de terror fue el que imperó.

\section{La tragedia}

El 24 de marzo de 1976, finalmente aconteció lo que muchos ya "imaginaban" que acontecería: las Fuerzas Armadas derrocaron el gobierno de Isabel Martínez de Perón. Lo que nadie imaginó es el grado de violencia de esta última dictadura ni el tiempo que duraría.

En lo que se refiere a las Escuelas de Trabajo o Servicio Social del país, los impactos fueron inmediatos aunque diferenciados. Algunas escuelas fueron intervenidas y otras fueron directamente cerradas ${ }^{18}$. La 
Escuela de Trabajo Social de Rosario, que en este período ya estaba adscripta a la Universidad, fue "clausurada ${ }^{19}$ por motivos de público conocimiento", luego de ser cercada e invadida por los militares. Parte de sus alumnos y profesores tuvieron que pasar a la clandestinidad, otros al exilio y todos fueron atravesados por el miedo al igual que el resto de la sociedad.

Ante la clausura del único espacio de formación que existía en la ciudad, se coartó la posibilidad de continuar con la formación académica y quienes no habían terminado de cursar sus estudios, tuvieron que trasladarse a la ciudad de Santa Fe para poder culminarlos. Fue a esta escuela donde se llevaron algunos de los archivos de la Escuela de Rosario, que en un acto simbólico fueron devueltos en 2005.

En fin, el advenimiento de la última dictadura militar trajo como una de sus consecuencias la interrupción del proceso de formación de los estudiantes, los graduados y los docentes. Significó la falta de nuevos profesionales en el mercado de trabajo, de actualización y capacitación de sus egresados, así como también la reducción de los espacios de inserción profesional durante un decenio; evidentemente ningún tipo de discusión teórica era posible en este período. Sin embrago, y paradójicamente, la necesidad de reorganización colectiva surgió en esta coyuntura.

Frente a la clausura del espacio formativo y a pesar del difícil escenario social que se vivía, (en donde todo "circulaba clandestinamente"); un grupo de trabajadores sociales comenzó a reunirse, retomando la conocida estrategia de la época del onganiato ${ }^{20}$, de organización $\mathrm{y}$ trabajo en pequeños grupos, como forma de poder compartir las experiencias, de debatir lo que acontecía en la profesión como en la sociedad, claro está, que esto se realizaba en un primer momento en una semiclandestinidad y en sus propias casas. Fue en estos grupos donde germinó la idea y la necesidad de formar la Asociación de Asistentes Sociales de Rosario, teniendo como primer objetivo continuar con la capacitación y formación de los trabajadores sociales, y para ello se entendía que era indispensable la reapertura de la Escuela de Trabajo Social, que finalmente fue abierta en 1986, ya dentro de un gobierno democrático y no más adscripta a la universidad, sino plenamente en el ámbito de la universidad. La Asociación de Asistentes Sociales de Rosario fue fundada en 1980 (aunque desde 1978 venían trabajando), procurando articularse a nivel local con otras Asociaciones Profesionales, a nivel nacional con otras organizaciones de la categoría profesional e inclusive a nivel internacional, lo que posibilitó la realización de un curso de capacitación con colegas chilenos. Otro de los objetivos que se propuso esta Asociación fue crear el Colegio de Trabajadores Sociales de Rosario, encargado de fiscalizar el ejercicio profesional, que finalmente fue concretado en 1987.
Es decir, tanto el Colegio de Profesionales de Trabajo Social, como la Escuela de Trabajo Social, dependiente de la Facultad de Ciencia Política y Relaciones Internacionales de la Universidad Nacional de Rosario, pudieron ser creadas durante el régimen democrático y a partir de la Asociación de Asistentes Sociales.

\section{La democratización}

Luego de la derrota de la guerra de Malvinas, en 1982, y con el advenimiento de la democracia en 1983, teniendo como marco una importante participación popular, y sobre todo, saliendo de los "destrozos" que había dejado la dictadura, se vislumbraba la posibilidad de vivir con un cierto grado de "civilidad", considerando que uno de los medios para alcanzarla sería la ampliación de las políticas públicas, con un carácter de universalidad, para posibilitar la recuperación de los niveles de protección social, de empleo y de condiciones de vida de tiempos atrás. A la esperanza democrática se sumaban los juzgamientos que se habían realizado a las Juntas de Comandantes, siendo la Argentina el único país de América del Sur que realizaba estos juicios. Sin embargo, "las ilusiones democráticas" poco duraron. La calidad de vida de los argentinos no mejoró, la hiperinflación comenzó a corroer los salarios de los trabajadores y la desocupación generó los saqueos. A esto se le sumó que, durante el gobierno de Alfonsín, se decretaron las leyes de Punto Final ${ }^{21}$ y Obediencia Debida y durante el gobierno de Menem el Indulto a los militares. Estas leyes son hoy en día cuestionadas en la justicia.

El ambiente de participación popular y, como ya dijimos, de civilidad, junto al proceso de redemocratización política, fue sumamente importante para la profesión, ya que logró recuperar la formación profesional. Esto quiere decir, que posibilitó retomar las discusiones teóricas y políticas que habían sido cortadas una década atrás. La inserción de las Escuelas de Trabajo Social dentro del ámbito de la universidad fue sumamente positiva, ya que creó una posibilidad de debate con las otras profesiones, con las otras disciplinas, y sobre todo, porque se vio ante la necesidad de producir conocimiento y de construirlo a partir de investigaciones, lo que generó equipos de investigación, y sobre todo un salto teórico. Es un camino a ser consolidado.

En lo que se refiere a la organización de la categoría profesional, la articulación que ésta consiguió luego de la dictadura también fue positiva. Los espacios académicos se organizaron, a nivel nacional, a partir de la Federación Argentina de Unidades Académicas de Trabajo Social (FAUATS), contando con el incansable trabajo de profesores y alumnos, ya que los recursos con los que cuenta la Federación son sumamente escasos. Hoy en día esta Federación cambió de nombre, pasando a denominarse Asociación Argentina de Formación Académica en Trabajo Social (AAFTS). 
También fue importante la reorganización que se dio en el interior de los colegios profesionales de Trabajo Social, ya que además de reglamentar el ejercicio profesional y controlar la matrícula, trabajó articuladamente con los espacios de formación profesional, posibilitando la consolidación de ambos espacios.

Es importante destacar la política de postgrados que se viene fomentando en la Argentina ${ }^{22}$, que posibilita el crecimiento de las producciones teóricas e investigaciones.

\section{Desafíos}

Pasadas tres décadas del golpe de Estado de 1976, la sociedad argentina se ha modificado, así como el Trabajo Social. Evidentemente se ha realizado un salto cualitativo, sea en la producción de conocimientos, sea en el ámbito de la actuación profesional; aunque no podemos dejar de mencionar que éstos avances se han realizado a "contramarcha de la Historia", dentro de un escenario que es adverso a los proyectos sociales que buscan la emancipación humana. Es un escenario donde se implanta como modelo de gestión social ${ }^{23}$ el proyecto neoliberal, cuyo interés principal es la "salud del mercado", desmantelando el Estado, privatizando el Estado, proponiendo un programa de políticas públicas con una connotación cada vez más privadas o como diría Yazbek (2004, p. 71) con una refilantropización de las políticas públicas.

Esa refilantropización, se construye a partir de referenciales no politicos, es decir, de 'iniciativas morales de ayuda al necesitado, que no producen derechos y no son judicialmente reclamables'. En resumen, se despolitizan y despublicizan en la sociedad, los padrones de protección social, dentro de un contexto de precarización, no apenas de las políticas públicas, sino del propio orden jurídico-político, aunque con resistencias.

En la década del 90, gran parte de la sociedad argentina fue sometida a la pobreza extrema y vio degradarse su calidad de vida. El agravamiento de la cuestión social llegó a formas impensables en la sociedad argentina; algunas décadas atrás esto era inimaginable.
Las condiciones de trabajo de los trabajadores sociales, también sufrieron el impacto de las transformaciones que se produjeron en el interior del Estado. En este sentido se viene produciendo una des-reglamentación del trabajo, con mayores grados de precarización, de inestabilidad, lo que sin duda causa una inseguridad en el trabajo. Aquí le cabe un papel fundamental a

los colegios profesionales del país, encargados de fiscalizar el ejercicio profesional, ya que las "barreras" entre las profesiones son cada vez más tenues. También deben fiscalizar las condiciones en que se desarrolla el trabajo profesional.

En lo que se refiere a la universidad, no tenemos duda del proceso de consolidación de las carreras de grado y del importante nivel académico que estas han alcanzado; al mismo tiempo deberán ser fortalecidas las políticas de postgrados, con calidad académica, fundamentadas en investigaciones y no meramente instrumentales. Somos conscientes de que, a la universidad, cada vez más, se le quiere dar un papel instrumental, productivista y utilitarista; uno de los desafíos que nos son puestos, es luchar contra esta imposición, construyendo una universidad verdaderamente democrática. En este camino sería necesario pensar en ejes que articulen las carreras de grado y de postgrados así como los ejes de las diferentes Escuelas de Trabajo Social de todo el país.

Otro desafío es fortalecer los núcleos y centros de investigación que existen en el país; ello es un desafío doble, teniendo en cuenta las condiciones adversas en que se desarrollan las tares de investigación, acompañados de un escaso financiamiento y la falta de una política seria y consistente. Así mismo es importante pensar en políticas de capacitación continuada para el colectivo profesional, trabajando de forma mancomunada la universidad y las organizaciones profesionales.

En este camino consideramos fundamental una política de integración con Latinoamérica y especialmente en el marco del Mercosur, sea con una política de cooperación entre centros académicos, sea entre las organizaciones profesionales. Recordemos que fue a mediados de la década del 60 , cuando comenzó a diseñarse un trabajo social "latinoamericano", y éste continúa siendo un desafío, claro que en otro contexto, y con otras bases teóricas. Evidentemente mucho se ha avanzado desde entonces, pero todavía quedan muchos desafíos. 
En el plano de la vida social, seguimos reafirmando nuestro compromiso con los sectores subalternos, con las clases con las que trabajamos cotidianamente; esto se traduce en la lucha por democratizar el acceso a la educación, a la cultura, a los servicios sociales, es decir, por la ampliación de las políticas públicas, con carácter de universalidad, con garantía real de los derechos sociales, que también se traduce en la construcción de una cultura política verdaderamente democrática. La historia es una página abierta y el rumbo que ella tome dependerá de lo que colectivamente las clases sociales organizadas realicen y esto dependerá de la lucha de clases. Como ya anunciamos en el comienzo de este trabajo, la historia nos tiene que servir como lección.

La revolución social del siglo XIX no puede sacar su poesía del pasado, sino solamente del porvenir.

Carlos Marx

\section{Recebido em 30.04.2006. Aprovado em 21.06.2006.}

\section{Referencias}

ALAYÓN, N. Antecedentes del Trabajo Social en la Argentina (primera aproximación). Cuaderno n. 16, v. II, Lima, Peru: Ed. CELATS, 1978

ANGUITA, E.; CAPARRÓS, M. La voluntad. Una historia de la militancia revolucionaria en Argentina. Tomo I: 1966-1973. Buenos Aires: Grupo Editorial Norma, 1998.

ANTOGNAZZI, I. La Lucha Armada en la Estrategia Política del PRT-ERP(1965-76). In: ANTOGNAZZI, I.; FERRER, R. (Comps.). Del Rosariazo a la democracia del 83. Escuela de Historia, Facultad de Humanidades y Artes, Univ. Nacional de Rosario, Rosario, Argentina, 1995.

BALVE, B.; MURMIS, M.; MARIN, J. C. et al. Lucha de calles, lucha de clases. Elementos para su análisis (Córdoba 1971-1969). Buenos Aires: Ed. la Rosa Blindada, 1973.

BENJAMÍN, W. O narrador. Considerações sobre a obra de Nikolai Leskov. In: OBRAS escolhidas. Magia e técnica, arte e política. São Paulo: Ed. Brasiliense, 1994.

CASTRONOVO, R. Los procesos de revisión, evaluación y reformulación de los proyectos de formación profesional de los trabajadores sociales argentinos. 1999. Disertación (Maestría en Servicio Social)- Pontifícia Univ. Católica, São Paulo, 1999.

HOBSBAWN, E. Historia del siglo XX. Barcelona, España: Ed. Crítica, 1997.

MOLJO, Shirly J. Formas organizativas del colectivo profesional: las experiencias de la Asociación de Asistentes Sociales de Rosario y del Colegio de profesionales de Trabajo Social, segunda circunscripción, Pcia de Santa Fe. Boletín Especial del día del Trabajador Social, Rosario, Argentina, Ed. Espacio \& Colegio Profesional de Trabajo Social, 2005.

MOLJO, Carina B. Trabajadores sociales en la historia. Una perspectiva transformadora. Argentina: Ed Espacio, 2005.

NETTO, J. P. Ditadura e Serviço Social. Uma análise do Serviço Social no Brasil pós 64. São Paulo: Cortez, 1996.

. O Serviço Social e a tradição marxista. Serviço Social \& Sociedade, São Paulo: Cortez, n. 30, p. 89-102, abr. 1989.

OLLIER, M. M. La creencia y la pasión. Privado, público y político en la izquierda revolucionaria. Buenos Aires: Ed. Ariel, 1998.

ROMERO, L. A. Breve historia contemporánea Argentina. Buenos Aires: Ed. Fondo de Cultura Económica, 1998.

ROZAS PAGAZA, M. La intervención profesional en relación con la cuestión social. El caso del Trabajo Social. Argentina: Ed. Espacio, 2001.

SIGAL, S. Intelectuales y poder en Argentina. La década del sesenta. Buenos Aires: Ed. Siglo XXI, 2002.

YAZBEK, M. C. Cuestión Social y Trabajo Social: desafíos profesionales para el Trabajo Social en Brasil. Revista Escenarios, n. 8, p. 69-74, Buenos Aires: Ed. Espacio, 2004.

\section{Notas}

1 Si bien existe una amplia bibliografía sobre la dictadura en la Argentina, así como algunos libros que trabajan sobre la historia del Trabajo Social, desconocemos la existencia de trabajos, que la aborden desde la perspectiva de los atores.

2 Las investigaciones a las que nos referimos son: Moljo, Shirly J. (2005) y Moljo, Carina (2005).

3 Aunque en momentos históricos diferentes, los más expresivos fueron la revolución cubana, la revolución china y el gobierno de Allende en Chile. Al respecto véase Anguita e Caparrós (1998), Hobsbawn (1997) y Ollier (1998), entre otros.

4 Como citan innúmeros autores, entre ellos Netto, (1989) era un marxismo sin Marx, ya que no se recurría al autor, sino a los manuales que circulaban en la época.

5 Cf. Hobsbawn, 1997.

6 Cf. Rozas Pagaza, 2001.

7 Servicio Social Tradicional "la práctica empirista, reiterativa, paliativa y burocratizada' de los profesionales, que medida 'por una ética liberal-burguesa' y cuya teleología 'consiste en la corrección - desde un punto de vista claramente funcionalista - de resultados psicosociales considerados negativos o indeseables, sobre el sustrato de una concepción (abierta o velada) idealista y/o mecanicista de la dinámica social, siempre presupuesta al ordenamiento capitalista de la vida como un dato factual ineliminable" (NETTO, 1996, p. 117-118). 
8 La ideología del desenvolvimiento fue defendida por organismos internacionales, en el contexto de la guerra fría y a partir de la creación de las Naciones Unidas en 1945. El subdesarrollo era asociado a los valores tradicionales, que fundamentalmente se encontraban en la cultura rural y que se basaban en un modo de producción donde tenía preponderancia lo agrario; ya el desarrollo era asociado a los valores modernos que se encontraban preponderantemente en las ciudades, siendo las industrias el modelo de producción apropiado.

9 Denominado por algunos como Tarsitano por ser su primer director y por otros como Bolívar, por ubicarse en la calle Bolívar.

10 El grupo argentino que se nucleaba en la editora y librería ECRO, había surgido en el Instituto de Serviço Social antes mencionado y fue conocido como la generación de 65 , ya que fue uno de los primeros grupos en "incorporarse" al proceso de reconceptuación. Cuando este grupo comenzó, seguía la línea inaugurada por Pichón Riviere, el Esquema Conceptual Referencial Operativo (ECRO).

11 Véase al respecto: Antognazzi (1995); Balve, Murmis, Marin et al. (1973).

12 Recordemos que los golpes militares y los regímenes democráticos se alternan en la Argentina desde la década del 1930, cuando fue derrocado el presidente Irigoyen. El golpe de estado de 1966 fue presidido por Onganía, que fue destituido luego de los levantamientos populares, asumiendo Levigston y luego Lanusse, hasta que se retornó a la democracia en 1973, que vigoró hasta 1976, momento en el que se produjo el último golpe de estado en Argentina. En 1983 se retomó la vida democrática.

13 La noche de la intervención fue conocida como la Noche de los Bastones Largos, cf. Romero (1998).

14 En algunos casos se constituyó en una vía de ingreso a las organizaciones político-militares.

\section{Así como en Latinoamérica}

16 Esta Asociación fue creada en el marco del V Congreso Panamericano de Servicio Social, realizado en Perú.

17 El artículo también muestra la expansión y crecimiento de las universidades nacionales durante este período, ya que fueron creadas 12 universidades nacionales, 2 universidades provinciales fueron transformadas en nacionales y se crearon 2 nuevas universidades provinciales. En 1975 existían en la Argentina 25 Universidades Nacionales,7 Universidades Provinciales y 23 universidades privadas.

18 Conforme consta en el estudio realizado por Castronovo (1999, p. 8): "De un total de cuarenta y cinco Escuelas de Trabajo Social que existían en ese momento, catorce fueron cerradas o suspendidas en su funcionamiento.” Recordando que, la universidad ya había sido intervenida en 1975.

19 La Escuela de Trabajo Social fue clausurada el 20 de octubre de 1976. Administrativamente la misma funcionó hasta 1977, año en que se decretó la Resolución No 3957/77 a través de la cual sus funciones administrativas, también, entraron en suspensión. El Poder Ejecutivo de la Provincia de Santa Fe dictó la resolución 593/76 por el cual se dispone la clausura temporaria de la Escuela de Servicio Social de Rosario por motivos que fueren de público conocimiento. Luego el Gobernador de la Provincia de Santa Fe, Vicealmirante Jorge Desimoni, firmó el decreto $\mathrm{N}^{\mathrm{o}} 3362 / 76$ por el cual se habilita a los estudiantes de Rosario a poder finalizar sus estudios como alumnos libres en la Escuela de la ciudad de Santa Fe; disponiéndose al mismo tiempo el cierre definitivo de la Escuela de Trabajo Social de Rosario, refrendado por el Ministro de Bienestar Social, Comodoro Raúl Fraga.

20 Referente al gobierno de Onganía.

21 En 1985 comenzaron los juicios a los militares, que finalizaron ese mismo año. Durante los mismos se revelaron las atrocidades cometidas por los militares; la Comisión Nacional para la Desaparición de Personas (Conadep), cuyo texto final fue publicado como Nunca Más, creada por el presidente Alfonsín y presidida por Ernesto Sábato, tuvo un papel fundamental en este proceso, junto con los organismos de Derechos Humanos, en especial las Madres de Plaza de Mayo. El fallo condenó a los ex comandantes y se propuso continuar la acción contra los demás responsables de las “operaciones". En 1985 fue promulgada la ley de Punto Final que daba un plazo de dos meses para las citaciones judiciales. Luego del levantamiento militar en Campo de Mayo y de Semana Santa de 1987, encabezada por el teniente coronel Aldo Rico, fue decretada la ley de Obediencia Debida, dejando sin responsabilidades a quienes habían recibido órdenes... En 1990, durante el gobierno de Menem, se decretó el Indulto, y de esta manera quienes habían sido juzgados y condenados, volvieron a quedar "libres". Sin embargo, actualmente algunos de ellos siguen siendo juzgados por la "apropiación ilegal de menores" y dichos crímenes no se inscriben dentro de las leyes ni del Indulto antes mencionados, ni de los acuerdos internacionales firmados por la Argentina. También esta siendo discutida la legalidad de las leyes de Punto Final y Obediencia Debida, siendo que varios jueces la han decretado inconstitucional. Lo que demuestra que la dictadura, aún es una herida abierta.

22 En este sentido, vale la pena recordar la fundamental importancia que han tenido los trabajadores sociales brasileños, especialmente el Programa de Posgraduación de la PUC/SP (Pontificia Universidad Católica de San Pablo), que al firmar un convenio de cooperación internacional con la Universidad Nacional de La Plata, posibilitaron la formación de los primeros Magísteres y Doctores en Trabajo Social, que a su vez permitieron continuar con la capacitación en el interior de las diferentes unidades académicas.

23 Espacio privilegiado de actuación profesional.

\section{Shirly J. Moljo}

smoljo@hotmail.com shirly@arnet.com.ar

Universidad Nacional de Rosario

Escuela de Trabajo Social

Pcia. de Santa Fe

2000 Rosario, Provincia de Santa Fe

Argentina 
A 30 años del golpe militar en Argentina: aproximaciones a la historia del Trabajo...

\section{Carina B. Moljo}

carinamoljo@hotmail.com

Universidade Federal de Juiz de Fora

Faculdade de Serviço Social

Bairro Cidade Universitária

Juiz de Fora - Minas Gerais

CEP: 36036-330

\section{Comunicado}

A Revista Katálysis comunica com pesar o falecimento da professora Shirly Josefina Moljo, ocorrido em 18 de agosto de 2006, ao fechamento desta edição. 